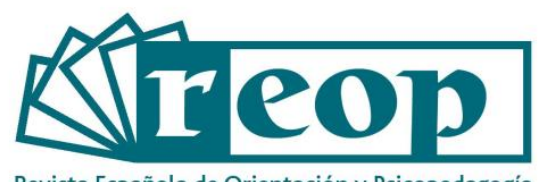

Revista Española de Orientación y Psicopedagogía

\title{
EL SISTEMA DE ORIENTACIÓN UNIVERSITARIA EN FINLANDIA: IDENTIFICACIÓN DE BUENAS PRÁCTICAS APLICABLES AL CONTEXTO ESPAÑOL
}

\section{THE UNIVERSITY GUIDANCE SYSTEM IN FINLAND: IDENTIFICATION OF GOOD PRACTICES APPLICABLE TO THE SPANISH CONTEXT}

\author{
Camino Ferreira ${ }^{1}$ \\ Universidad de León. Facultad de Educación. Departamento de Psicología, Sociología y Filosofía. \\ León, Área de Métodos de Investigación y Diagnóstico en Educación, España
}

\section{RESUMEN}

El propósito de este articulo es analizar, desde una aproximación documental, cuál es la oferta de servicios y actividades innovadoras en el contexto de orientación de las universidades públicas finlandesas. Este estudio está enmarcado en el paradigma cualitativo de investigación, identificando (a) qué servicios de orientación y asesoramiento ofrecen a sus estudiantes y (b) qué actividades innovadoras desarrollan en el ámbito de la orientación que puedan aplicarse al contexto español, mejorando nuestro sistema de orientación universitaria. Se han analizado las trece universidades finlandesas mediante la importación de la información de las páginas webs $(n=125)$ al software profesional para el análisis de datos cualitativos MAXQDA 2018. Partiendo de este análisis previo, así como de la consulta a expertos en orientación en la Universidad de Turku (Finlandia), se identificaron buenas prácticas del sistema de orientación universitaria español, seleccionando aquellas con mejores beneficios para los estudiantes y mayor transferibilidad y proyección internacional a nivel institucional. Los principales resultados obtenidos reflejan una tendencia en Finlandia a promover acciones dirigidas al bienestar de los estudiantes desde una unidad común e incluir la orientación como parte intrínseca del well-being de la universidad. Existen asimismo comités de igualdad y bienestar de los estudiantes para abordar estas dos cuestiones, aspecto que se podría trasladar a las universidades españolas. Por último, se destaca

${ }^{1}$ Correspondencia: Camino Ferreira Correo-e: camino.ferreira@unileon.es 
la transferibilidad de los planes personalizados de estudio para los estudiantes, la coordinación y la interacción entre los recursos existentes de orientación, y una mayor flexibilidad en las adaptaciones a realizar a los estudiantes.

Palabras clave: Universidad, orientación, servicios universitarios, servicios de orientación finlandeses, buenas prácticas.

\begin{abstract}
The purpose of this article is to analyse, from a documentary approach, which is the offer of innovative services and activities in the context in which guidance is developed in Finnish public universities. This study is framed within the qualitative paradigm of research, identifying (a) what guidance and counselling services they offer to their students and (b) what innovative activities they develop in the field of guidance that can be applied to the Spanish context, improving our university guidance systems. The thirteen existing universities in Finland have been analysed by importing the websites $(n=125)$ into the professional software for the analysis of qualitative data MAXQDA 2018. Starting from this previous analysis as well as from the consultation with experts in guidance at the University of Turku, good practices transferable to the Spanish university guidance system were identified, selecting those with the best benefits for students, greater transferability, and international projection at an institutional level. The main results obtained offer a tendency in Finland to promote actions aimed at the well-being of students from a common unit and to include guidance as an intrinsic part of the well-being of the university, existing also committees on equality or well-being of students, an aspect that could be transferred to Spanish universities. The transferability of Personal Study Plans for students, the coordination and interaction between existing guidance resources, and greater flexibility in the arrangements to be made with students are also highlighted.
\end{abstract}

Key Words: university, guidance, university services, Finnish guidance services, good practices.

\title{
Cómo citar este artículo:
}

Ferreira, C. (2021). El sistema de orientación universitaria en Finlandia: Identificación de buenas prácticas aplicables al contexto español. Revista Española de Orientación y Psicopedagogía, 32(1), 7-29. https://doi.org/10.5944/reop.vol.32.num.1.2021.30737

\section{Introducción}

La realidad que presenta la orientación universitaria actualmente se caracteriza por ser muy heterogénea, especialmente si nos centramos en analizar los servicios que se ofrecen y en las actuaciones que desarrollan en orientación (Pérez Cusó y Martínez Juárez, 2015). En España, los servicios de orientación comenzaron en los años setenta con la institucionalización de esta a través de los Centros de Orientación e Información de Empleo (COIE). Su evolución ha ido 
transformándose, abarcando más aspectos de la orientación, especialmente en su vertiente académica, y adaptándose al Espacio Europeo de Educación Superior. Actualmente estos servicios se focalizan más en la atención académica y presentan un carácter eminentemente puntual y terapéutico, desarrollando funciones informativas y de asesoramiento principalmente a nivel individual (Pérez Cusó y Martínez Juárez, 2015).

La actual Ley Orgánica 4/2007, de 12 de abril, por la que se modifica la Ley Orgánica 6/2001, de 21 de diciembre, de Universidades no menciona la orientación en su texto, puesto que fue incorporada como parte de los derechos de los estudiantes en la Ley Orgánica 6/2001, de 21 de diciembre, de Universidades. Este derecho se materializa en la orientación sobre las actividades que afecten a los estudiantes (artículo 46.c), así como el asesoramiento por parte de profesores y de tutores (artículo 46.e). Sin embargo, es en el Real Decreto 1791/2010, de 30 de diciembre, por el que se aprueba el Estatuto del Estudiante Universitario, donde se desarrolla ampliamente la orientación universitaria, no solo garantizándola como derecho de los estudiantes (artículos 7-10), sino también promoviéndola a través de programas de orientación que mejoren sus transiciones, integración y formación (artículo 7.a); en el ámbito vocacional, académico y profesional (artículo 7.f). Además, los dos principales hitos del Estatuto son la inclusión del capítulo V sobre la tutoría, en el que diferencia entre tutorías de titulación, de materia o asignatura y, por primera vez, para estudiantes con discapacidad; y el capítulo XV relativo a la atención al estudiante universitario donde menciona la existencia de los servicios de atención al estudiante que ofrezcan orientación en (artículo 65.3): elección y reformulación de estudios; metodologías de trabajo y formación en estrategias de aprendizaje; itinerarios formativos y salidas profesionales; actividades de formación a lo largo de la vida; asesoramiento psicológico y en materia de salud; igualdad de trato entre mujeres y hombres.

En la práctica, el sistema de orientación universitaria se caracteriza por presentar dificultades y retos en la coordinación e integración de todos los servicios, debido principalmente a la falta de recursos humanos y materiales necesarios para poder aunar sinergias (Pérez Cusó y Martínez Juárez, 2015). Uno de los ejemplos se concreta en el rol que tiene el profesorado en el ámbito de la orientación. El profesorado universitario ha venido asumiendo recientemente funciones a nivel educativo que superan los límites tradicionales de "instructores" para ejercer funciones de "orientadores", y en muchos casos, no disponen de las estrategias y herramientas requeridas para que puedan ejercerlas con éxito (San Román et al., 2015).

Centrándonos en la organización del sistema de orientación, observamos que en la Universidad española continúan con la estela de su origen, predominando los servicios especializados, en concreto en orientación profesional y orientación académica. Sin embargo, en los últimos años se han producido esfuerzos por converger los servicios existentes hacia unidades que aglutinen todas las especialidades. Un ejemplo de ello son las Unidades de Orientación de Centro como instrumentos para la orientación a nivel universitario. Estos servicios permanentes específicos, que podemos encontrar en universidades como la Universidad de Granada, cuentan con personal especializado y están completamente integrados en la estructura organizativa de la institución. Su ámbito de actuación cubre todas las actividades de orientación de forma sistemática y coordinada en los tres ámbitos de acción de la orientación mediante un modelo de funcionamiento por programas (Villena et al., 2013). Lo cierto es que, a pesar de estos esfuerzos por unificar servicios, el panorama es distinto y refleja la existencia de los Servicios de Información y Orientación Universitaria (SIOU) junto con los COIE, y otro tipo de servicios que intentan cubrir la dimensión personal de la orientación como, por ejemplo, los Servicios de Orientación Psicopedagógica, Gabinetes de Atención Psicológica o las Oficinas de Atención a la Diversidad (Biencinto et al., 2014).

Aunque la orientación ha tenido un origen vocacional, esta dimensión se ha ido ampliando para cubrir diversos ámbitos necesarios para lograr una orientación completa de los estudiantes universitarios. Así, en algunos estudios se han diferenciado como elementos de la orientación: orientación personal y académica de los alumnos, tutoría, orientación familiar, orientación para el 
tránsito entre etapas, orientación profesional y atención a la diversidad (Velaz-de-Medrano et al., 2012) o aprendizaje, atención a la diversidad, orientación académica y profesional, y prevención y desarrollo personal (Del Rincón Igea y Bayot Mestre, 2008). De forma sintética, podemos entender que el concepto de orientación se compone de tres áreas de actuación fundamentales, denominado Triángulo de la Orientación, el cual incluye la orientación profesional, la académica y la personal (Saúl et al., 2009). Centrándonos en esta última área, se ha observado que la dimensión personal en la universidad ha estado ligada a la acción tutorial con el objetivo de potenciar el desarrollo integral de los estudiantes desde dos vertientes, una más informativa sobre intereses y expectativas en los estudios y otra sobre asuntos más privados e íntimos (Haya Salmón et al., 2013). En el sistema de orientación universitaria, los servicios menos extendidos, e incluso conocidos por los estudiantes universitarios, han sido aquellos relacionados con el ámbito personal (Saúl et al., 2009).

Algunos procesos de acreditación europeos incluyen aspectos de orientación y apoyo a los estudiantes como elementos que proporcionan una Educación Superior de calidad (Ferreira et al., 2014; Lairio y Penttinen, 2006). En el caso de España, en el Documento Marco de la Agencia Nacional de Evaluación de la Calidad y Acreditación (ANECA) que incluye los criterios para la evaluación para la renovación de la acreditación de títulos oficiales de Grado y Máster Universitario (Programa ACREDITA) publicados en 2019, se incluye como aspecto objeto de valoración: los servicios de apoyo y orientación académica, profesional y para la movilidad puestos a disposición de los estudiantes una vez matriculados se ajustan a las competencias y modalidad del título y facilitan el proceso de enseñanza-aprendizaje. La ANECA Ileva considerando la orientación en sus criterios y directrices desde 2006, sin embargo, como se indica en la última publicación ofrecida, las dimensiones académica y profesional están contempladas, existiendo un vacío todavía sobre la dimensión personal de la orientación a pesar de que el Estatuto del Estudiante Universitario de 2010 así la recoge.

Los expertos consideran que se debería de materializar mejor la orientación como un factor de calidad de la institución (González López y Martín Izard, 2004) con el objetivo de facilitar el seguimiento y la mejora de la atención a los estudiantes universitarios. Existen algunas propuestas paralelas que abogan por incluir la orientación dentro de los procesos de evaluación (Ibarra Sáiz y Rodríguez Gómez, 2010), utilizar la tutoría académica como espacio para obtener feedback sobre estas acciones orientadoras y de integralidad formativa (López Gómez, 2017; Rodríguez-Hoyos et al., 2015), o utilizar un sistema de indicadores propio en la atención y el apoyo a los estudiantes con discapacidad (Ferreira et al., 2014). En cualquier caso, la universidad debe reflexionar y conocer qué necesidades de orientación presentan sus estudiantes y cuáles van cambiando para dar una respuesta de calidad y aumentar, por ende, su satisfacción, así como su éxito en su paso por la universidad (García Félix et al., 2014). Actualmente, las principales mejoras deben ir centradas en mejorar la comunicación con los estudiantes y egresados, potenciar la colaboración intrauniversitaria y fomentar el desarrollo de relaciones interuniversitarias y de alianzas con instituciones externas (Martínez Gómez, 2008).

Existen diversos estudios que han analizado los servicios de orientación en otras universidades como Francia, Estados Unidos, Canadá, Reino Unido, así como dentro de nuestro país (Flores Buils et al., 2012; Manzanares Moya, 2013), poniendo de manifiesto las diferencias que hay entre los distintos sistemas de orientación dependiendo del país analizado y su comparación con España. Entre ellos, destaca Finlandia por disponer de una buena reputación y calidad a nivel educativo, y de un sistema avanzado en lifelong guidance (Euroguidance, 2019; OECD, 2017); y se caracteriza por ofrecer acciones dirigidas principalmente al bienestar del estudiante como parte inherente a la misión de la universidad (Zarei et al., 2010). Partiendo de las necesidades identificadas y la oportunidad de transferir buenas prácticas de otros contextos en la prestación de actuaciones de orientación en la universidad española, en este estudio se analiza el sistema de apoyo y orientación a estudiantes universitarios en las universidades públicas finlandesas, identificando propuestas sobre las oportunidades de mejora y su aplicación al contexto español. 


\section{Método}

\section{Diseño y procedimiento}

El presente estudio se aborda desde un enfoque cualitativo-interpretativo. La metodología del trabajo adoptada se basa en el análisis de contenido, caracterizada por ser un método sistemático que permite examinar en detalle un fenómeno, en este caso la orientación, a partir de las principales fuentes de datos disponibles (Kettunen y Tynjälä, 2018; Rapley, 2014). Las páginas web institucionales de las universidades finlandesas han sido la principal fuente de análisis de datos, puesto que permiten la difusión de estos servicios y presentan flexibilidad y facilidad de actualización (García Félix et al., 2014). Además, son la principal y más extendida vía de información sobre los servicios de orientación entre los estudiantes (Martínez Gómez, 2008), facilitando la rápida localización y visibilidad del servicio tanto en las webs institucionales como en el propio organigrama de la institución.

El análisis de los contenidos de estas webs se ha realizado mediante procedimientos tecnológicos específicos que permiten el manejo de grandes volúmenes de información, lo que aumenta la validez de los resultados y su relevancia para la toma de decisiones. El procedimiento seguido ha sido: (1) selección de las universidades finlandesas a analizar, (2) identificación de los sitios web de las universidades y de la información sobre los servicios de orientación a los estudiantes, (3) importación web y análisis de la información recogida a través del software MAXQDA para el análisis de datos, y (4) revisión del proceso de codificación y redacción de los resultados.

Cabe señalar que este estudio se basa en un análisis de contenido, tomando información de documentos y fuentes descriptivas, no respecto a las acciones en sí, sino en base al marco legal existente en España y a las webs institucionales que se analizan en Finlandia.

\section{Muestra}

Según la Agencia Nacional de Educación de Finlandia, este país tiene dos tipos de Instituciones de Educación Superior, todas ellas públicas: las Universidades $(n=13)$ y las Universidades de Ciencias Aplicadas (UAS) ( $n=23$ ). En este estudio, sólo se han seleccionado las universidades, puesto que están centradas en la investigación y en la formación (estando más cerca del concepto de universidad española). Todas ellas ofrecen estudios a nivel de grado y máster e incluyen programas de doctorado, cubriendo más campos de conocimiento con respecto a las UAS. Este otro tipo de institución está muy orientado profesionalmente y conectado con la vida laboral y el desarrollo regional. Por lo tanto, las universidades analizadas han sido: la Universidad de Aalto, la Escuela de Economía de Hanken, la Universidad de LUT, la Universidad de Tampere, la Universidad de Finlandia Oriental, la Universidad de Helsinki, la Universidad de Jyväskylä, la Universidad de Laponia, la Universidad de Oulu, la Universidad de Turku, la Universidad de Vaasa, la Universidad Åbo Akademi y la Universidad de las Artes de Helsinki. Esta última 
universidad ha sido incluida en el análisis, pero no se han encontrado servicios de orientación debido a la naturaleza de esta.

\section{Recogida y análisis}

La identificación de la información a analizar se ha realizado a través de la búsqueda de los dos términos clave en inglés referidos a la orientación según el Tesauro de ERIC: guidance y counselling. Este estudio se centra en los servicios específicos de orientación, eliminando de la muestra aquellos dirigidos a informar $u$ orientar sobre otros aspectos tales como la internacionalización, administración y registro o espiritualidad. Se han llevado a cabo distintas estrategias para asegurar la validez interna de este diseño de investigación cualitativa, utilizando indicadores de baja inferencia (descripciones detalladas y literales de la información recogida), registrando de forma mecánica los datos a través del colector de sitios web del software MAXQDA y realizando varias rondas de búsqueda de información para verificar posibles pérdidas de información. Los datos se basan en textos disponibles con una amplia y suficiente información en inglés (ver ejemplo en Figura 1).

\section{Figura 1}

Ejemplo de tipo de documento analizado en el programa MAXQDA

\begin{tabular}{|l|l|}
\hline Visor de documento: Lifelong Learning at the University of Turku (Página 1/2) \\
\hline
\end{tabular}

Fuente: Documento analizado de la Universidad de Turku. 
Para la identificación y registro de la información se tuvieron en cuenta los siguientes criterios sobre cada institución y sus servicios de orientación con el fin de seleccionar la información más fiable relativa a las acciones y funciones que ofrecen en orientación:

- Universidad.

- Nombre del servicio.

- Visibilidad dentro de la web institucional.

- Organización dentro de la universidad.

- Áreas de intervención.

- Objetivos.

- Profesionales involucrados.

- Actividades específicas.

- Colaboración con otros servicios.

El número total de documentos web importados al software y analizados ha sido de 125 (ver Tabla 1). Del análisis de la información han resultado tres grandes categorías: profesionales implicados $(n=9)$, objetivos y funciones de las actividades de orientación $(n=19)$, y dimensiones de la orientación $(n=35)$, estableciéndose un total de 63 categorías y subcategorías (ver Anexo l). Las dimensiones han sido organizadas en función de su ámbito siguiendo la estructura de Eurydice: orientación académica, orientación profesional y asesoramiento psicológico. El número de segmentos codificados ha sido de 673.

\section{Tabla 1}

Documentos importados y segmentos codificados por universidad finlandesa

\begin{tabular}{lcccc}
\hline \multicolumn{1}{c}{ Universidad } & Acrónimo & $\begin{array}{c}\text { Número de } \\
\text { estudiantes* }\end{array}$ & $\begin{array}{c}\text { Documentos } \\
\text { importados }\end{array}$ & $\begin{array}{c}\text { Segmentos } \\
\text { codificados }\end{array}$ \\
\hline Universidad de Aalto & AYY & 17452 & 18 & 110 \\
Escuela de Economía de Hanken & HANKEN & 2500 & 10 & 40 \\
Universidad de LUT & LUT & 5217 & 7 & 39 \\
Universidad de Tampere & UTA & 13169 & 10 & 55 \\
Universidad de Finlandia Oriental & UEF & 15636 & 12 & 77 \\
Universidad de Helsinki & UH & 31245 & 12 & 50 \\
Universidad de Jyväskylä & JYU & 13813 & 6 & 45 \\
Universidad de Laponia & LaY & 4340 & 5 & 38 \\
Universidad de Oulu & OY & 12619 & 8 & 36 \\
Universidad de Turku & UTU & 16142 & 19 & 92 \\
Universidad de Vaasa & VYY & 4962 & 7 & 41 \\
Universidad Åbo Akademi & $\AA$ A & 5580 & 11 & 50 \\
\hline \multicolumn{1}{c}{ Total } & & & $\mathbf{1 2 5}$ & $\mathbf{6 7 3}$ \\
\hline
\end{tabular}

Fuente: Elaboración propia. * Últimos datos disponibles de mayo de 2019 en Statista. 
Una vez analizado el sistema de apoyo y orientación a estudiantes universitarios en las universidades públicas finlandesas, se han identificado propuestas sobre las oportunidades de mejora y su aplicación al contexto español. Para este objetivo, se ha partido del análisis previo, así como de la consulta a tres expertos en orientación (dos orientadores y un docente con tareas de mentoría) en la Universidad de Turku, institución donde se ha desarrollado el estudio financiado, siendo la técnica de muestreo no probabilística por conveniencia y discrecional. Los expertos consultados a través de entrevistas individuales desarrollan tareas de orientación profesional, mentoría y orientación académica con los estudiantes finlandeses. La consulta se centró en el análisis de aquellas actuaciones en orientación con mejores beneficios para los estudiantes y con mayor transferibilidad y proyección internacional a nivel institucional desde su perspectiva.

\section{Resultados}

Sistema de apoyo y orientación a estudiantes universitarios en las universidades públicas finlandesas

El sistema de orientación finlandés atiende, al igual que en España, la orientación desde las tres principales dimensiones: académica, profesional y personal. Esta última dimensión es identificada en Finlandia como la dimensión psicológica de la orientación. La forma en la que ofrecen los servicios y actuaciones difiere de una universidad a otra dentro del país, pero en todos los casos, la orientación ofrecida se caracteriza por ser confidencial y de carácter tanto proactivo como reactivo. De forma general, la organización de los servicios de orientación a estudiantes depende principalmente de los Servicios Estudiantiles, los Servicios de Educación/Aprendizaje o de la Oficina de Asuntos Estudiantiles. Sin embargo, en algunas universidades esta organización presenta diferencias. En unos casos, estos servicios incluyen las tres dimensiones; en otros, la académica y la psicológica están bajo la misma unidad o servicio (p.ej. AYY), dependiendo incluso los servicios psicológicos de los de orientación académica (p.ej. Hanken). El factor común en este sistema es que los servicios de orientación profesional se encuentran muy bien delimitados, como ocurre también en España, aunque en Finlandia destacan por su coordinación con el resto de los servicios, incluyendo asimismo psicólogos de estudio y planificación profesional.

En relación con los objetivos y funciones de los servicios de orientación en las universidades finlandesas (ver Figura 2), se observa que estos se centran fundamentalmente en el apoyo (25\%) y la ayuda $(23,5 \%)$ a los estudiantes. Otras acciones que también están muy relacionadas con la orientación son proporcionar, ofrecer, discutir y asegurar (entre un 5,9-13,2\%) herramientas, habilidades o un plan de estudio personal, entre otros. Existen en un tercer lugar, funciones destinadas a organizar, potenciar, aconsejar, asistir y guiar (menos del $4 \%$ ) a fin de promover el éxito de los estudiantes en la universidad. 


\section{Figura 2}

Porcentaje de objetivos principales que ofrecen los servicios de orientación en las universidades finlandesas

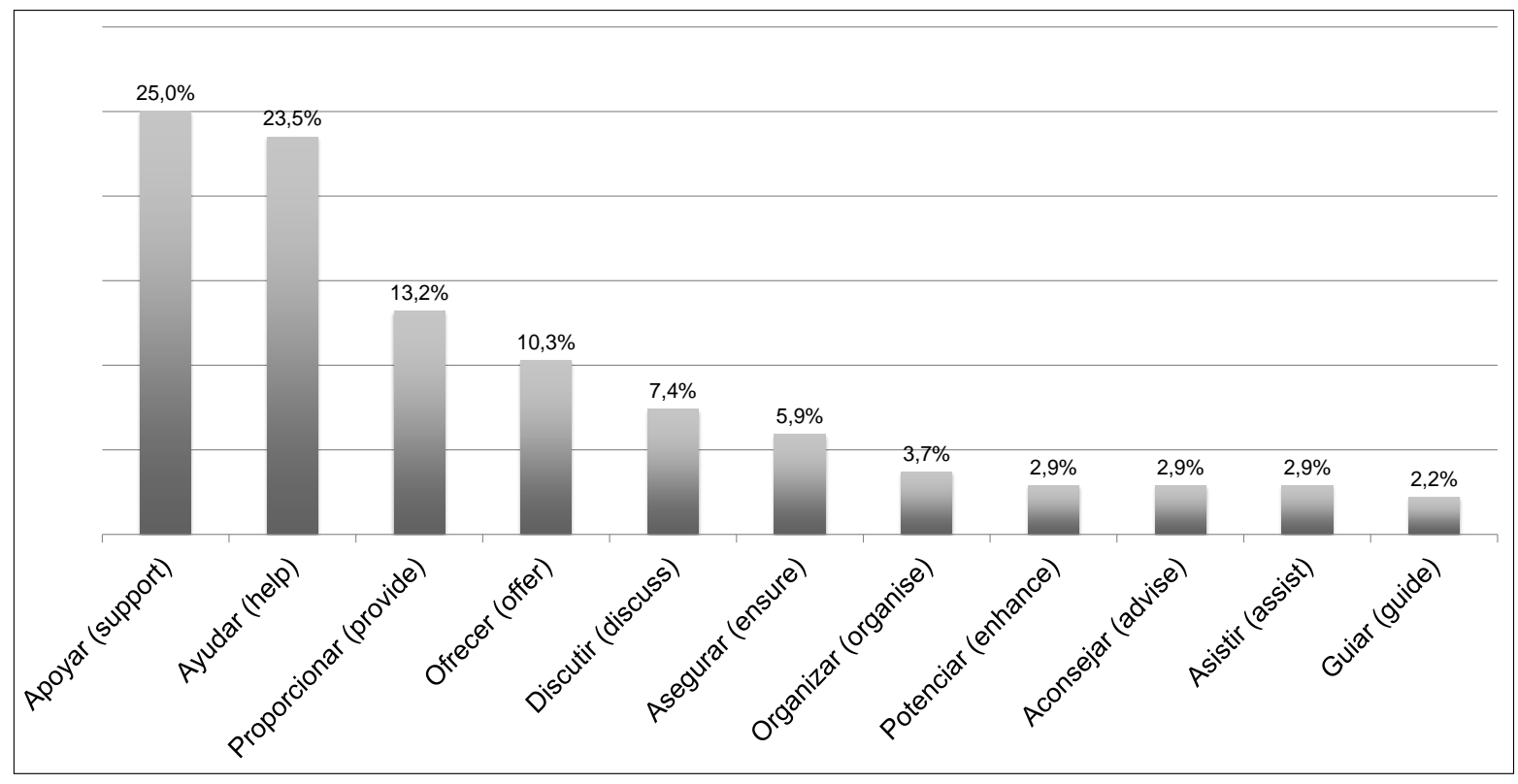

Fuente: Elaboración propia.

Se han identificado diferentes perfiles profesionales involucrados en estos servicios para cada área de la orientación. Sin embargo, desde las universidades se pone especial énfasis en la colaboración de todos los orientadores y profesores de la universidad en tareas de orientación, especialmente aquellas relacionadas con la identificación y tratamiento de situaciones inapropiadas por parte de la comunidad como el acoso o la intimidación hacia otras personas. Los profesionales identificados en los servicios de orientación y asesoramiento ( $n=56$ segmentos) se han agrupado en función de sus responsabilidades y dedicación. De esta manera, diferenciamos la tutoría, el asesoramiento, la psicología, la accesibilidad y el acoso.

En las actividades relacionadas con la tutoría, se diferencian dos perfiles profesionales: el alumno tutor (student tutor) y el profesor tutor (teacher tutor). Por un lado, los alumnos tutores ayudan a los nuevos estudiantes a familiarizarse con la cultura y la sociedad finlandesa, la vida universitaria y los asuntos prácticos, así como con asuntos relacionados con los estudios. Los alumnos tutores solicitan este papel de forma voluntaria y reciben formación para su tarea. Por otro lado, el profesor tutor es un consejero que apoya y supervisa a los estudiantes, orientándolos regularmente en sus estudios, pero también en lo que se refiere a su futura profesión y otras cuestiones de orientación laboral. Existe la figura del coordinador de tutores para asegurarse del buen funcionamiento de este sistema de profesores tutores.

En el asesoramiento como tal, podemos destacar al orientador (counsellor), responsable de la planificación de los estudios, así como de la orientación laboral de los estudiantes. Esta figura se puede delimitar en función de la orientación que ofrece, siendo académica y dirigida a la planificación de los estudios en las diferentes etapas académicas; o siendo laboral, centrándose en temas relacionados con planes de vida laboral, solicitud de empleo o planificación de carrera.

En el campo de la psicología, todas las universidades analizadas tienen la misma figura profesional: el psicólogo del estudio (study psychologist). Este profesional trabaja entre la orientación académica y el trabajo en salud mental. Incluso, en algunos casos se centran también 
en la orientación laboral, existiendo la figura del psicólogo de planificación de la carrera. Los estudiantes pueden solicitar su ayuda en caso de que estén preocupados por la consecución de sus estudios o tengan que mejorar sus habilidades académicas, la motivación, la gestión del tiempo, el bienestar o cualquier otro problema que les afecte en sus estudios.

En el ámbito de la accesibilidad, dos universidades finlandesas disponen de un profesional especializado en ello (accessibility planning officer) (UTA, UTU). Estos profesionales están disponibles en caso de que los estudiantes necesiten ayuda individual o tengan condiciones especiales, como por ejemplo enfermedad, lesión o discapacidad; ayudando también a los profesores a abordar y tratar estas circunstancias.

Para la prevención y el tratamiento de los casos de acoso, algunas universidades (VYY, AYY, UEF) tienen una persona de contacto (harassment contact person) en esta materia generalmente dentro de la delegación de estudiantes de la universidad, cuya función es proporcionar orientación a los estudiantes que han sufrido acoso o mala conducta en la comunidad académica y ayudarles a resolver la situación.

Si analizamos la presencia de estos perfiles profesionales en las universidades finlandesas, en la Figura 3 se puede observar que la principal figura en la orientación es la que realiza acciones específicas de tutoría $(41,2 \%)$, seguido por las de asesoramiento $(25,4 \%)$ y las psicológicas $(21,6 \%)$. En menor medida, se encuentran las relacionadas con el acoso y la accesibilidad (5,9\%).

\section{Figura 3}

Porcentajes de los principales ámbitos de actuación de los profesionales involucrados en los servicios de orientación universitaria finlandeses

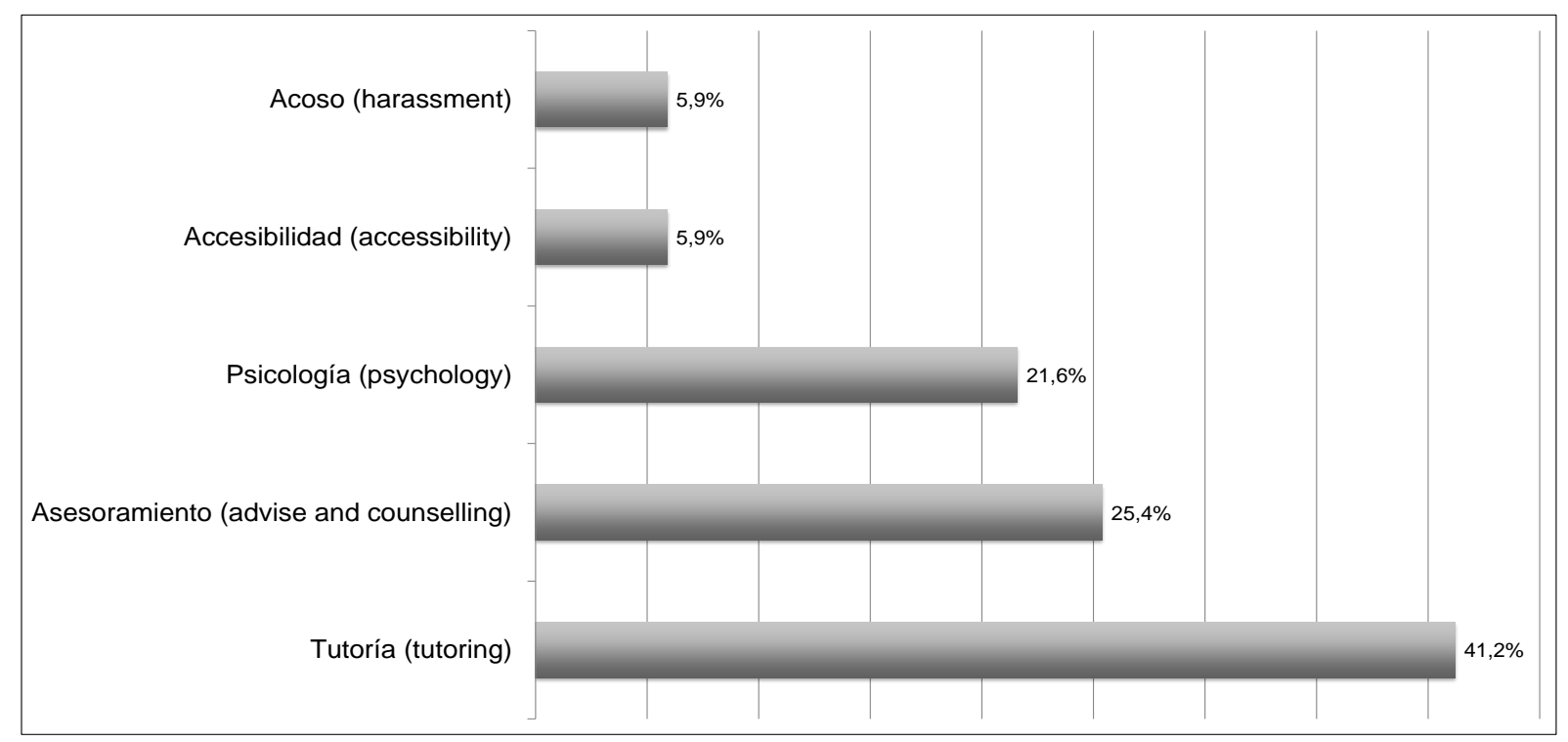

Fuente: Elaboración propia.

En el sistema de orientación universitaria finlandés, todas las dimensiones de este se encuentran bajo la misma "unidad" o "servicio" que se suele denominar servicios de orientación y asesoramiento (Guidance and Counselling Services), incluyendo tanto la orientación académica, como el estudio psicológico y el asesoramiento sobre la vida laboral. En algunas universidades este concepto se denomina Guidance Corner (UH) o Student Life Wellbeing (JYU). Sin embargo, 
cada una de las dimensiones ofrece actuaciones concretas y propias. En la Figura 4 se puede observar una visualización de los códigos asignados a los documentos analizados por cada universidad, detallándose las medidas específicas ofrecidas por cada una de ellas en función de las tres dimensiones de orientación. La intensidad y el tamaño del nodo refleja el cálculo proporcional de los segmentos codificados en relación con el total de la matriz. De esta forma, por universidad, se observa que la mayor información sobre orientación se encuentra en AYY, UEF y UTU. Si nos centramos en las dimensiones de la orientación, se observa un peso mayor en actividades de orientación académica $(n=58)$, seguido de las de asesoramiento psicológico $(n=48)$ $y$, por último, en orientación profesional $(n=30)$. Las actividades de orientación a las que se concede más importancia se concentran en la orientación en el estudio, la accesibilidad y adaptaciones especiales; vida laboral y búsqueda de empleo; y bienestar de los estudiantes, así como el cuidado de la salud y cuestiones de igualdad de trato y diversidad.

En relación con la orientación académica, las universidades finlandesas cuentan con una orientación en este ámbito muy bien organizada y dirigida a asegurar que los estudiantes alcancen sus metas académicas. Esta orientación suele depender de los Servicios Estudiantiles y Académicos o de la Oficina de Asuntos Académicos y Estudiantiles; y está muy relacionada con la orientación psicológica en la realización de adaptaciones a los estudiantes, así como en cuestiones de motivación y habilidades en el estudio. Los orientadores ofrecen apoyo en los estudios en orientación individual y preventiva, planificación, permanencia y finalización, compensación y reconocimiento, protección jurídica y cuestiones de accesibilidad.

La orientación profesional depende del Servicio de Orientación Profesional (Career Service) y está orientado a dos acciones principales: la planificación de la carrera y la búsqueda de empleo. Sus objetivos son ayudar a los estudiantes universitarios a desarrollar el autoconocimiento y tomar decisiones relacionadas con sus carreras, mejorar sus aptitudes para la vida laboral y la búsqueda de empleo, y definir sus aspiraciones y oportunidades profesionales. Por ello, la orientación profesional en Finlandia no es sólo un apoyo en la búsqueda de empleo, sino que también proporciona a los estudiantes herramientas para encontrar respuestas y aclarar sus ideas sobre su futura carrera y sus aspiraciones profesionales, así como sobre sus competencias y puntos fuertes personales. Estos servicios trabajan tanto con estudiantes y egresados como con empleadores, y están disponibles desde el comienzo de los estudios hasta un año después de la graduación; tal y como se indica: "las habilidades para la vida laboral se desarrollan constantemente a lo largo de sus estudios, a veces sin que se den cuenta". Algunas universidades, como la UF, ofrecen sesiones de e-counselling para proporcionar acciones de orientación profesional más flexibles.

Con relación a la dimensión personal o psicológica, se ofrece orientación principalmente individual en diferentes áreas: motivación, aprendizaje, confianza en sí mismo, ansiedad, autorregulación, dificultades de aprendizaje, estrés y tensión, sentirse atrapado en los estudios (no progresar), habilidades de estudio, estrategias de afrontamiento, establecimiento de objetivos, incorporación a los estudios después de una larga pausa e incluso elección de carrera. También se encuentran muy relacionados con los problemas de accesibilidad como entorno mental, estando disponibles de forma gratuita, voluntaria y confidencial para los estudiantes universitarios. Este asesoramiento psicológico se realiza normalmente en el marco del bienestar de los estudiantes y es ofrecido por los Servicios de Psicología del Estudio (Study Psychologist Services) como parte de los Servicios de Aprendizaje. Los psicólogos del estudio (especializados en la psicología del aprendizaje, el asesoramiento y la orientación) se encargan de evaluar las necesidades de desarrollo del estudiante en aproximadamente cinco sesiones. De esta forma, "el estudiante y el psicólogo del estudio se esfuerzan por encontrar soluciones juntos, desarrollar habilidades de estudio y nuevas formas de pensar y actuar, mejorando así las condiciones de estudio". Además, en algunas universidades, los estudiantes también tienen la posibilidad de participar en asesoramientos en grupo. 


\section{Figura 4}

Visualización de los códigos asignados a los documentos analizados por cada universidad

\begin{tabular}{|c|c|c|c|c|c|c|c|c|c|c|c|c|c|c|}
\hline \multicolumn{2}{|c|}{ Sistema de códigos } & AYY & HANK... & LUT & \multirow[t]{2}{*}{ UTA } & UEF & \multirow[t]{2}{*}{ UH } & \multirow[t]{2}{*}{ JY } & \multirow[t]{2}{*}{ LaY } & \multirow[t]{2}{*}{ OY } & \multirow[t]{2}{*}{ UTU } & \multirow[t]{2}{*}{ VYY } & \multirow[t]{2}{*}{ ÀA } & \multirow{2}{*}{$\begin{array}{c}\text { SUMA } \\
0\end{array}$} \\
\hline$\nabla$ & Dimensiones de orientación & & & & & & & & & & & & & \\
\hline$\nabla$ & e Orientación académica & & $\mathbf{\square}$ & & & $\mathbf{\square}$ & $\square$ & $\square$ & $\mathbf{\square}$ & $\square$ & & $\square$ & & 58 \\
\hline & ๑ Orientación en el estudio & - & & $\square$ & $\square$ & $\square$ & & & $\square$ & $\mathbf{\square}$ & - & $\mathbf{a}$ & & 20 \\
\hline & ๑ Tutoría académica & & & - & - & - & $\square$ & $\mathbf{\square}$ & & $\mathbf{\square}$ & - & & & 10 \\
\hline & ๑ Técnicas y habilidades de estudio & $\mathbf{\square}$ & & $\mathbf{\square}$ & & a & - & & & & & & & 11 \\
\hline & $\odot$ Accesibilidad y adaptaciones especiales & $\square$ & & & $\mathbf{\square}$ & $\square$ & $\mathbf{a}$ & & & & $\square$ & & $\mathbf{\square}$ & 26 \\
\hline & $\odot$ Plan personal de estudio & & & & $\square$ & & & & $\square$ & $\mathbf{\square}$ & $\square$ & & $\mathbf{\square}$ & 19 \\
\hline $\boldsymbol{\nabla}$ & ○ Orientación profesional & - & $\square$ & 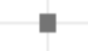 & घ & $\mathbf{\square}$ & $\mathbf{a}$ & $\mathbf{\square}$ & $\square$ & घ & $\mathbf{\square}$ & - & $\mathbf{\square}$ & 30 \\
\hline & $\odot$ Búsqueda de empleo & $\square$ & $\square$ & $\square$ & & $\square$ & & $\mathbf{\square}$ & $\mathbf{\square}$ & & $\mathbf{a}$ & & & 15 \\
\hline & ๑ Planificación de la carrera & . & $\mathbf{\square}$ & & - & $\mathbf{\square}$ & & & $\mathbf{\square}$ & & - & & & 11 \\
\hline & $\odot_{\bullet}$ Adaptación del CV & & & $\mathbf{\square}$ & & & & $\square$ & & & - & & & 6 \\
\hline & $\odot_{\bullet}$ Vacantes, prácticas y tutorías & & $\mathbf{\square}$ & & & 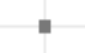 & & $\square$ & & & - & - & & 10 \\
\hline & ○ Conocimiento de sí mismo y habilidades propias & & & $\mathbf{a}$ & & $\mathbf{\square}$ & & & & & & & & 5 \\
\hline & $\odot$ Vida laboral & $\square$ & $\square$ & $\square$ & & $\mathbf{\square}$ & & $\square$ & & & & - & & 15 \\
\hline $\boldsymbol{\nabla}$ & Asesoramiento psicológigo & $\square$ & $\mathbf{\square}$ & $\square$ & $\mathbf{a}$ & $\square$ & $\Pi$ & $\mathbf{a}$ & $\square$ & $\square$ & $\mathbf{a}$ & $\mathbf{\square}$ & $\square$ & 48 \\
\hline & $\ominus_{\ominus}$ Cuidado de la salud & $\cdot$ & घ & $\square$ & & $\square$ & - & $\mathbf{\square}$ & - & & & $\square$ & $\mathbf{\square}$ & 12 \\
\hline & $\odot$ Bienestar & $\cdot$ & - & & & $\mathbf{\square}$ & - & - & $\square$ & & - & घ & $\square$ & 15 \\
\hline & $\odot$ Igualdad de trato y diversidad & $\square$ & & & & $\square$ & - & & & & $\square$ & & $\square$ & 12 \\
\hline & $\odot$ Condición para estudiar: autoconocimiento y recursos & & & & & & - & & $\mathbf{\square}$ & & & & & 5 \\
\hline & $\odot_{A}$ Ayuda con el abuso de sustancias & & & & - & - & - & & & & & & & 3 \\
\hline & ๑ Gestión de habilidades psicológicas & & & & & & & & & & & $\square$ & & 8 \\
\hline & 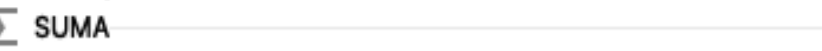 & 57 & 22 & 20 & 28 & 43 & 26 & 21 & 19 & 12 & 42 & 25 & 24 & 339 \\
\hline
\end{tabular}

Fuente: Elaboración propia. 


\section{Propuestas del sistema de orientación universitaria finlandés sobre las oportunidades de mejora y su aplicación al contexto español}

Partiendo del análisis realizado y de las recomendaciones de los expertos consultados, el siguiente propósito de este estudio es identificar buenas prácticas en las universidades finlandesas en materia de orientación como propuestas y oportunidades de mejora en su aplicación al contexto español. En el Anexo II se puede observar el modelo del sistema de orientación universitaria finlandés en el que se incluyen las buenas prácticas incluidas en el mismo, así como la relación entre las actividades desarrolladas en materia de orientación. Se han identificado las siguientes actuaciones innovadoras y propias del sistema finlandés que pueden ser agrupadas en función de las áreas de orientación.

En el ámbito de la orientación académica, el Plan Personal de Estudio (Personal Study Plan, PSP) es la actuación más destacable. Se trata de un Plan personalizado e individualizado para cada estudiante realizado de forma conjunta entre el estudiante y su tutor. Cada profesor tutor se encarga de aproximadamente 5-10 estudiantes, reservando tiempo de su horario para este trabajo. Este Plan, realizado al inicio de los estudios y aprobado por el tutor, se establece sobre la base del plan de estudios a cursar por el estudiante, así como de sus objetivos personales, actualizándose durante la realización de los estudios. El PSP contempla tres aspectos fundamentales: (1) los estudios necesarios, ayudando en su planificación a largo plazo; (2) el progreso del estudio, ayudando a evaluar el desarrollo de competencias; y (3) la planificación, ayudando en la gestión del tiempo personal. Algunos aspectos previos a considerar en el momento de realizar el PSP son: objetivos y recursos personales del estudiante, asignaturas, estudios de idiomas y cursos de formación, condiciones y momentos de estudio o posibilidad de participar en un programa de movilidad. Asimismo, se hace hincapié en el reconocimiento del aprendizaje previo (Recognition of Prior Learning), teniendo en cuenta que las competencias adquiridas previamente por los estudiantes en la educación formal básica, experiencia laboral, actividades organizativas, etc., podrían sustituir algunos resultados de aprendizaje de cursos y/o módulos de estudio específicos.

Dentro de la orientación académica, todas las universidades finlandesas inciden en la accesibilidad y las adaptaciones especiales para los estudiantes. La accesibilidad significa que la universidad está libre de barreras, es segura y saludable y que todos sus servicios y actuaciones son accesibles para todos en su entorno físico, social y mental. Por lo tanto, esta accesibilidad es especialmente importante para los estudiantes con discapacidad o dificultades de aprendizaje. En el Estatuto del Estudiante Universitario español se indica que en cada universidad se fomentará la creación de Servicios de Atención a la comunidad universitaria con discapacidad, mediante el establecimiento de una estructura que haga factible la prestación de los servicios requeridos por este colectivo. En el caso de las universidades finlandesas, el colectivo a atender no se queda en la presentación de un certificado de discapacidad, sino que también cubre otras dificultades derivadas de problemas de ansiedad, salud mental, dificultades de aprendizaje en lectura, escritura y matemáticas (ofreciendo evaluaciones gratuitas para su diagnóstico) o TDAH. En el caso de estudiantes que presenten temporalmente una enfermedad o lesión, el profesorado puede realizar los ajustes necesarios para apoyar su progreso académico y bienestar. No existe una lista común de adaptaciones, ya que dependen de las necesidades de cada estudiante, pero generalmente están relacionadas con adaptaciones metodológicas como la ampliación del tiempo o formas alternativas en la evaluación, y con la provisión de recursos técnicos de apoyo o asistente personal/intérprete. Estas adaptaciones no afectan al currículo, no modificándose los estándares académicos y los criterios de evaluación. En algunas universidades cuentan con un panel de expertos que ofrece sugerencias para la toma de decisiones de estas adaptaciones.

En la dimensión profesional, una de las acciones más importantes para ellos es conocer cómo es el mercado laboral. Un ejemplo específico es el Hanken International Talent (HIT), que ofrece a 
los estudiantes internacionales de alto rendimiento un acceso único a las empresas finlandesas que buscan fortalecer su ventaja competitiva a través de la internacionalización. Sin embargo, la fortaleza más destacable de la orientación profesional es la Red de Servicios de Orientación Profesional de las Universidades Finlandesas, la cual depende del Gobierno Finlandés y es conocida como Aarresaari. Esta red, formada por doce universidades, proporciona servicios de coordinación nacional en el sector de la orientación profesional, siendo parte de la estrategia nacional de educación en Finlandia para favorecer el éxito de los egresados. Sus temas de interés se centran en proporcionar a los estudiantes un nivel suficientemente alto de conocimiento de la vida y mercado laboral, promoviendo modelos eficaces de cooperación entre las universidades y la comunidad empresarial.

Algunas de las acciones de los servicios de orientación profesional están destinadas a que los estudiantes profundicen en el conocimiento sobre sí mismos y sus propias habilidades, identificando sus puntos fuertes y definiendo las habilidades necesarias para su perfil profesional. En este sentido, se ofrece formación centrada en el análisis de las ofertas de trabajo y la redacción de solicitudes de empleo adecuadas a sus necesidades y a las de los empleadores. Por último, también evalúan las habilidades en el empleo de los estudiantes, proporcionándoles retroalimentación sobre aquellas que necesitan ser mejoradas.

Como se ha mencionado anteriormente, la dimensión personal en Finlandia está muy extendida e implantada en la universidad dentro del well-being del estudiante. Algunas universidades como LaY disponen de un Well-being Committee para promover y apoyar el bienestar, la salud y la capacidad de estudio de los estudiantes. Además de ello, todos los servicios de asesoramiento psicológico de las universidades finlandesas tienen un vínculo con el Servicio Finlandés de Salud Estudiantil (Finnish Student Health Service, FSHS). Este servicio va más allá de lo que ofrecen los Servicios de Psicología del Estudio de las universidades y funciona en caso de situaciones de salud muy graves y complicadas como depresión, ansiedad, crisis, ataques de pánico, trastornos alimentarios, etc. Junto con este servicio, una de las mejores prácticas en la dimensión personal es Nyyti ry, una organización finlandesa sin ánimo de lucro que ofrece consejos y herramientas para promover la salud mental de los estudiantes a través de información y actividades sobre el bienestar mental.

Una parte importante de la dimensión personal es la gestión de habilidades psicológicas. Esta actuación se centra en la psicología del aprendizaje, el asesoramiento y la orientación, y el desarrollo de competencias. El orientador en este ámbito trabaja en la promoción de la motivación, la gestión del tiempo, la progresión de los estudios y el bienestar general, especialmente en temas relacionados con la ansiedad y el manejo del estrés.

Tres de las universidades finlandesas analizadas (UH, UEF, UTA) ofrecen un programa para estudiantes para el tratamiento de abuso de sustancias. En algunos casos, hay incluso una persona en cada facultad para tratar estos problemas, apoyando al estudiante y, si es necesario, poniéndose en contacto con el FSHS.

Por último, la mayoría de las universidades finlandesas (AYY, UEF, UH, UTU, ÅA) ofrecen apoyo a los estudiantes en casos de acoso. Cualquier tipo de comportamiento de acoso, trato inapropiado o intimidación está prohibido en estas universidades, especialmente en el proceso de enseñanza-aprendizaje o en actuaciones de orientación. Las universidades implican a toda la comunidad universitaria haciéndola responsable de asegurar una atmósfera de respeto y un entorno seguro, cómodo y libre de discriminación. En caso de acoso, los estudiantes pueden comunicarse con las personas de contacto de su universidad para discutir y tratar el tema, tomando medidas necesarias como la mediación y el seguimiento. Las universidades proporcionan instrucciones en caso de acoso visible para todos los estudiantes en su página web. Para abordar las cuestiones de igualdad, la UTU cuenta con una Comisión de Igualdad nombrada por el Rector, cuya tarea es avanzar y supervisar la aplicación y el desarrollo de la igualdad en la universidad. Esta actuación refleja la preocupación que existe en Finlandia sobre el acoso. Es 
destacable el programa nacional KiVA contra la intimidación desarrollado en UTU cuyo objetivo es reducir la intimidación y la victimización cambiando las respuestas de los espectadores que presencian estos actos a través de las experiencias vividas por ellos y de entornos de aprendizaje virtual (Yang y Salmivalli, 2015).

Como medida transversal y complementaria en orientación, algunas universidades finlandesas incluyen sistemas de evaluación y control propios. Por ejemplo, en AYY administran un cuestionario denominado: AllWell? study well-being questionnaire para mejorar el éxito de los estudiantes mediante la planificación de acciones que fomenten su bienestar, identificando algunos factores que influyen en el estrés y la carga de trabajo. Los resultados permiten desarrollar los programas y actuaciones de orientación y supervisión de estudios, la formación para profesores sobre la carga de trabajo de los estudiantes y el seguimiento del progreso y el bienestar de los estudiantes. Otro ejemplo se encuentra en UTU, donde disponen de un Sistema de Retroalimentación y Evaluación de Estudiantes sirviendo de referencia para el desarrollo y mejora de la orientación, entre otros servicios. Asimismo, se encuentra el sistema de datos Peppi, el cual es muy utilizado en el sector educativo finlandés para responder a las necesidades de las universidades a través de un sistema integral de administración de estudiantes.

\section{Conclusiones}

Este estudio recoge una descripción en profundidad sobre la orientación universitaria en Finlandia con el objetivo de poder implementar buenas prácticas en las universidades españolas en este ámbito. Por ello, este estudio no pretende hacer una comparación empírica del sistema de orientación español con este país nórdico, especialmente porque las diferencias existentes no sólo en el ámbito educativo, sino a nivel cultural y social, hacen que las acciones ofertadas y su organización tengan un origen y naturaleza propias.

El sistema de orientación se caracteriza por ser heterogéneo en las universidades. Esto ocurre también en Finlandia y en España. Sin embargo, hay líneas de organización similares, especialmente en los servicios de orientación profesional, los cuales tiene un carácter más independiente y presentan acciones equiparables. En el caso de la orientación académica y personal, estos servicios varían cualitativamente. Se han propuesto denominaciones y organizaciones que conlleven un sistema común integrado de orientación en la universidad española a través de un servicio de orientación universitaria como el "Servicio de Orientación Psicopedagógica y Profesional" (Flores Buils et al., 2012). Esta idea permitiría aprovechar los recursos ya existentes, mejorando el funcionamiento coordinado de los mismos e implicando a todos los agentes (Manzanares Moya, 2013; Suárez-Ortega et al., 2017). Esto ocurre en algunas universidades finlandesas donde la orientación parte de una misma unidad, concentrando los recursos existentes y facilitando al estudiante el punto de contacto y orientación en la universidad para cualquiera de las acciones que la integran. La clave es fomentar la coordinación y la interacción constante entre los recursos existentes en materia de orientación, buscando la interrelación entre los intereses académicos con la búsqueda de empleo, y teniendo en cuenta las aspiraciones del estudiante, sus características y necesidades personales. De esta manera, se ofrecería una fuente de información clara especialmente entre las conexiones (previas) de itinerarios formativos y las salidas profesionales (posteriores), aumentando la eficacia de los estudiantes (Skaniakos et al., 2019) y las oportunidades laborales (Palomares-Montero et al., 2019). En este sentido, se parte de una orientación global y "para todos", para concretarse en una 
orientación "para cada estudiante", profundizando en nuevas vías como la orientación online (Paterson et al., 2017).

En el contexto finlandés, el sistema de orientación universitaria se lleva a cabo gracias a la presencia de diferentes profesionales involucrados. El abanico de figuras identificadas es heterogéneo y amplio, destacando el perfil del tutor y del orientador, los cuales se encuentran en el sistema español. Sin embargo, existen asimismo otros perfiles que permiten la especialización de la orientación y atender cuestiones de bienestar con fines determinados como, por ejemplo, el alumno tutor, el psicólogo, la persona de contacto en caso de acoso o de accesibilidad. Si bien, estas funciones podrían recaer en figuras ya existentes y de gran tradición en España como es el tutor a través de los planes de acción tutorial (Pérez Cusó y Martínez Juárez, 2015), siempre y cuando se proporcione la formación y el espacio temporal para ello, especialmente en el caso de los estudiantes de nuevo ingreso (Lizalde et al., 2018). El propósito de las funciones de estos profesionales en el contexto español partiría desde los distintos niveles para atender las necesidades que integran las dimensiones de la orientación, favoreciendo la conexión entre los servicios y el resto de la estructura académica, y materializando el Estatuto del Estudiante Universitario en el que se explicita que las unidades y servicios de orientación deberán desarrollar sus funciones estrechamente conectadas y coordinadas con los sistemas de acción tutorial, las acciones de formación de tutores y el conjunto de programas y servicios de la universidad (artículo 65.2).

A lo largo de este estudio se han descrito buenas prácticas transferibles al sistema universitario de orientación en España. Entre ellas se destacan los planes personalizados de estudio para los estudiantes a través de las acciones como herramienta de apoyo para el buen desarrollo de los estudios y el bienestar de los estudiantes. Esta medida va más allá de la acción tutorial y permite realizar un seguimiento individualizado del estudiante principalmente a nivel académico, teniendo en cuenta su proyección laboral y las necesidades personales que pudieran afectarle en su paso por la universidad. Asimismo, aunque nuestro sistema de orientación ofrece adaptaciones a los estudiantes, especialmente a través de las unidades de atención a estudiantes con discapacidad, sería conveniente disponer de una mayor flexibilidad en las adaptaciones a realizar en caso de enfermedad o lesión temporal. Estos acuerdos flexibles tratan de ayudar a los estudiantes a lograr los resultados de aprendizaje esperados. En lo referente a las acciones de orientación profesional, estas deberían incluirse dentro de los planes de estudios universitarios (Izquierdo Rus y Farías Gragmena, 2018).

Los principales resultados obtenidos ofrecen una tendencia a promover acciones dirigidas al bienestar de los estudiantes desde una unidad común e incluir la orientación como parte intrínseca del well-being de la universidad (Zarei et al., 2010), existiendo asimismo comités de igualdad o bienestar de los estudiantes, incluso un panel de expertos en accesibilidad y adaptaciones especiales. Estas prácticas no son destacadas en los estudios sobre orientación universitaria y podrían trasladarse a las universidades españolas. En el contexto español, este tipo de dimensión personal o relacionada con aspectos psicológicos sigue siendo una dimensión desconocida y ambigua, incluyéndose como asesoramiento psicológico en la normativa, pero aún no explicitada en los procedimientos universitarios de calidad nacional (ANECA).

La efectividad de las acciones descritas desde distintos puntos de vista no ha sido objeto de estudio de este artículo, lo que limita su alcance. En este sentido, las líneas de avance investigador que sugieren estos resultados se centrarían en dos vías. Por un lado, en el estudio de la viabilidad de las buenas prácticas identificadas, así como en las recomendaciones de estas por parte de los principales interesados: los estudiantes. De esta forma, se completaría el estudio con otro tipo de recogida de datos que confirmen la información recogida en las webs institucionales. Por otro lado, dado que la dimensión personal o psicológica no está definida claramente en la universidad, sería interesante indagar tanto en la conceptualización como en las actuaciones específicas, necesarias y eficaces que ya se están desarrollando en algunas universidades. 


\section{Referencias bibliográficas}

ANECA (2006). Criterios y directrices para la acreditación de enseñanzas universitarias conducentes a títulos oficiales españoles de Grado y Máster. Agencia Nacional de Evaluación de la Calidad y Acreditación.

ANECA (2019). Documento Marco: Evaluación para la renovación de la acreditación de títulos oficiales de Grado y Máster Universitario (Programa ACREDITA). Agencia Nacional de Evaluación de la Calidad y Acreditación.

Biencinto López, C., García-García, M., Carpintero Molina, E. y Núñez Del Río, M. C. (2014). Servicios de Orientación Universitaria: visibilidad y propuestas de mejora. Revista de Orientación Educacional, 28(54), 15-30. http://www.roe.cl/index.php/roe/article/view/82

Del Rincón Igea, B. y Mestre, A. B. (2008). Resultados y propuestas de un servicio de orientación universitaria. Revista Española de Orientación y Psicopedagogía, 19(2), 138-148. https://doi.org/10.5944/reop.vol.19.num.2.2008.11415

Euroguidance (2019). Guidance System in Finland. https://www.euroguidance.eu/guidancesystem-in-finland

Ferreira, C., Vidal, J. y Vieira, M. J. (2014). Student Guidance and Attention to Diversity in the Processes of Quality Assurance in Higher Education. European Journal of Education, 49(4), 575-589. https://doi.org/10.1111/ejed.12098

Ferreira, C., Vieira, M. J. y Vidal, J. (2014). Sistema de indicadores sobre el apoyo a los estudiantes con discapacidad en las universidades españolas. Revista de Educación, 363, 412-444. https://doi.org/10.4438/1988-592X-RE-2012-363-193

Flores Buils, R., Gil Beltrán, J. M. y Caballer Miedes, A. (2012). Rol del servicio de orientación en universidades de Francia, EE.UU., Canadá, Reino Unido y España. Papeles Del Psicólogo, 33(2), 138-147. http://www.papelesdelpsicologo.es/pdf/2102.pdf

García Félix, E., Conejero Casares, J. A. y Diez Ruano, J. L. (2014). La entrada en la Universidad: un reto para la orientación académica. REDU. Revista de Docencia Universitaria, 12(2), 255280. https://doi.org/10.4995/redu.2014.5650

González López, I. y Martín Izard, J. (2004). La orientación profesional en la Universidad, un factor de calidad según los alumnos. Revista Española de Orientación y Psicopedagogía, 15(2), 299-315. https://doi.org/10.5944/reop.vol.15.num.2.2004.11635

Haya Salmón, I., Calvo Salvador, A. y Rodríguez-Hoyos, C. (2013). La dimensión personal de la tutoría universitaria. Una investigación cualitativa en la Universidad de Cantabria (España). Revista Española de Orientación y Psicopedagogía, 24(3), 98-113. https://doi.org/10.5944/reop.vol.24.num.3.2013.11247

Ibarra Sáiz, M. S. y Rodríguez Gómez, G. (2010). Los procedimientos de evaluación como elementos de desarrollo de la función orientadora en la universidad. Revista Española de 
$\begin{array}{llll}\text { Orientación } \quad y & \text { Psicopedagogía, } & \text { 21(2), }\end{array}$ https://doi.org/10.5944/reop.vol.21.num.2.2010.11558

Izquierdo Rus, T. y Farías Gragmena, A. J. (2018). Empleabilidad y expectativa de logro en la inserción laboral de los estudiantes universitarios. Revista Española de Orientación y Psicopedagogía, 29(2), 29-40. https://doi.org/10.5944/reop.vol.29.num.2.2018.23151

Kettunen, J. y Tynjälä, P. (2018). Applying phenomenography in guidance and counselling research. British Journal of Guidance and Counselling, 46(1), 1-11. https://doi.org/10.1080/03069885.2017.1285006

Lairio, M. y Penttinen, L. (2006). Students' career concerns: challenges facing guidance providers in higher education. International Journal for Educational and Vocational Guidance, 6, 143157. https://doi.org/10.1007/s10775-006-9107-z

Ley Orgánica 4/2007, de 12 de abril, por la que se modifica la Ley Orgánica 6/2001, de 21 de diciembre, de Universidades (Boletín Oficial del Estado, núm. 89, de 13 de abril de 2007, 16241-16260).

Ley Orgánica 6/2001, de 21 de diciembre, de Universidades (Boletín Oficial del Estado, núm. 307, de 24 de diciembre de 2001, BOE-A-2001-24515).

Lizalde Gil, M., Casanova López, O., Serrano Pastor, R. M. y Escolano Pérez, E. (2018). Plan de orientación universitaria para los estudiantes de nuevo ingreso. Programación de acciones y elaboración de materiales. Revista Española de Orientación y Psicopedagogía, 29(2), 41-54. https://doi.org/10.5944/reop.vol.29.num.2.2018.23152

López-Gómez, E. (2017). El concepto y las finalidades de la tutoría universitaria: una consulta a expertos. Revista Española de Orientación y Psicopedagogía, 28(2), 61-78. https://doi.org/10.5944/reop.vol.28.num.2.2017.20119

Manzanares Moya, A. (2013). Sistemas integrados de orientación. una propuesta para la articulación de estructuras y servicios a nivel local. Revista Española de Orientación y Psicopedagogía, 24(1), 62-77. https://doi.org/10.5944/reop.vol.24.num.1.2013.11271

Martínez Gómez, F. (Dir.) (2008). Análisis de los servicios de información, orientación y apoyo proporcionados por las universidades y utilización de los mismos por los universitarios. Programa de Estudios y Análisis Dirección General de Universidades (proyecto EA20070235). Fundación Universidad Empresa.

OECD (2017). PISA 2015 Results (Volume V): Collaborative Problem Solving, PISA. Paris: OECD Publishing. http://dx.doi.org/10.1787/9789264285521-en

Palomares-Montero, D., Chisvert-Tarazona, M. J. y Suárez-Ortega, M. (2019). Formación y orientación para el emprendimiento. Lo que dicen la bibliometría y los emprendedores noveles. Revista Española de Orientación y Psicopedagogía, 30(1), 131-149. https://doi.org/10.5944/reop.vol.30.num.1.2019.25198

Paterson, S. M., Laajala, T. y Lehtelä, P. L. (2017). Counsellor students' conceptions of online counselling in Scotland and Finland. British Journal of Guidance \& Counselling, 47(3), $292-$ 303. https://doi.org/10.1080/03069885.2017.1383357

Pérez Cusó, F. J. y Martínez Juárez, M. (2015). Tutoría universitaria y servicios de orientación: Dos realidades en un mismo contexto. Revista Electrónica Interuniversitaria de Formación Del Profesorado, 18(2), 177-192. https://doi.org/10.6018/reifop.18.2.219461 
Rapley, T. (2014). Los análisis de la conversación, del discurso y de documentos en Investigación Cualitativa. Ediciones Morata.

Real Decreto 1791/2010, de 30 de diciembre, por el que se aprueba el Estatuto del Estudiante Universitario (Boletín Oficial del Estado, núm. 318, de 31 de diciembre de 2010, BOE-A-201020147).

Rodríguez-Hoyos, C., Calvo Salvador, A. y Haya Salmón, I. (2015). La tutoría académica en la educación superior. Una investigación a partir de entrevistas y grupos de discusión en la Universidad de Cantabria (España). Revista Complutense de Educación, 26(2), 467-481. https://doi.org/10.5209/rev RCED.2015.v26.n2.43745

San Román, S., Vecina, C., Usategui, E., Del Valle, A. I. y Venegas, M. (2015). Representaciones Sociales y Orientación Educativa del Profesorado. Archivos Analíticos de Políticas Educativas, 23(128), 1-22. http://dx.doi.org/10.14507/epaa.v23.2088

Saúl, L. A., López-González, M. A. y Gutiérrez Bermejo, B. (2009). La orientación educativa en las universidades españolas: de la orientación laboral y vocacional a la atención psicológica. Acción Psicológica, 6(1), 7-15. https://doi.org/10.5944/ap.6.1.226

Skaniakos, T., Honkimäki, S., Kallio, E., Nissinen, K. y Tynjälä, P. (2019). Study guidance experiences, study progress, and perceived learning outcomes of Finnish university students. European Journal of Higher Education, 9(2), 203-218. https://doi.org/10.1080/21568235.2018.1475247

Suárez-Ortega, M., Sánchez-García, M. F. y García-García, M. C. (2017). Caracterización de buenas prácticas y necesidades de mejora en los servicios de orientación para el empleo. Revista Española de Orientación y Psicopedagogía, 27(3), 43-60. https://doi.org/10.5944/reop.vol.27.num.3.2016.18799

Velaz-De-Medrano Ureta, C., Blanco-Blanco, Á. y Manzano-Soto, N. (2012). Cobertura de necesidades de orientación y tutoría en la Educación Obligatoria: Estudio en nueve comunidades autónomas. Revista de Educación, número extraordinario, 138-173. https://doi.org/10.4438/1988-592X-RE-2012-EXT-210

Villena Martínez, M. D., Muñoz García, A. y Polo Sánchez, M. T. (2013). La Unidad de Orientación de Centro como instrumento para la Orientación Universitaria. REDU. Revista de Docencia Universitaria, 11(2), 43-62. https://doi.org/10.4995/redu.2013.5566

Yang, A. y Salmivalli, C. (2015). Effectiveness of the KiVa antibullying programme on bully-victims, bullies, and victims. Educational Research, 57(1), 80-90. https://doi.org/10.1080/00131881.2014.983724

Zarei, E., Shaikhi Fini, A. A. y Khajehzadeh Fini, H. (2010). A comparison of Effect of group counselling methods, behavioural, cognitive, and cognitive- behavioural to reduce students Test anxiety in the University of Hormozgan. Procedia - Social and Behavioural Sciences, 5, 2256-2261. https://doi.org/10.1016/..sbspro.2010.07.445

Fecha de entrada: 21 noviembre 2019

Fecha de revisión: 12 mayo 2020

Fecha de aceptación: 21 mayo 2020 


\section{Anexos}

\section{Anexo I}

Sistema de códigos resultante del análisis cualitativo

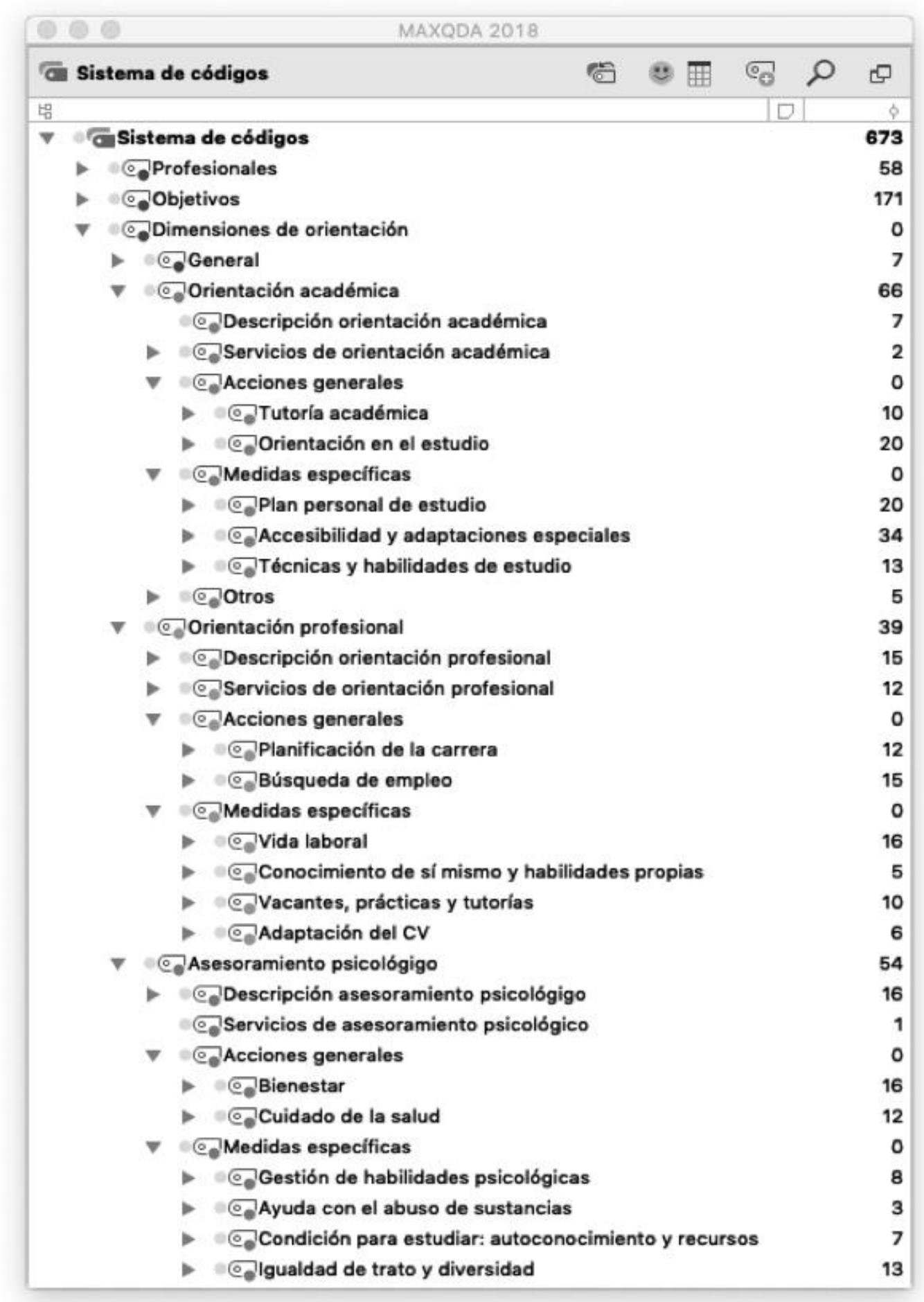




\section{Anexo II}

Modelo del sistema de orientación universitaria finlandés

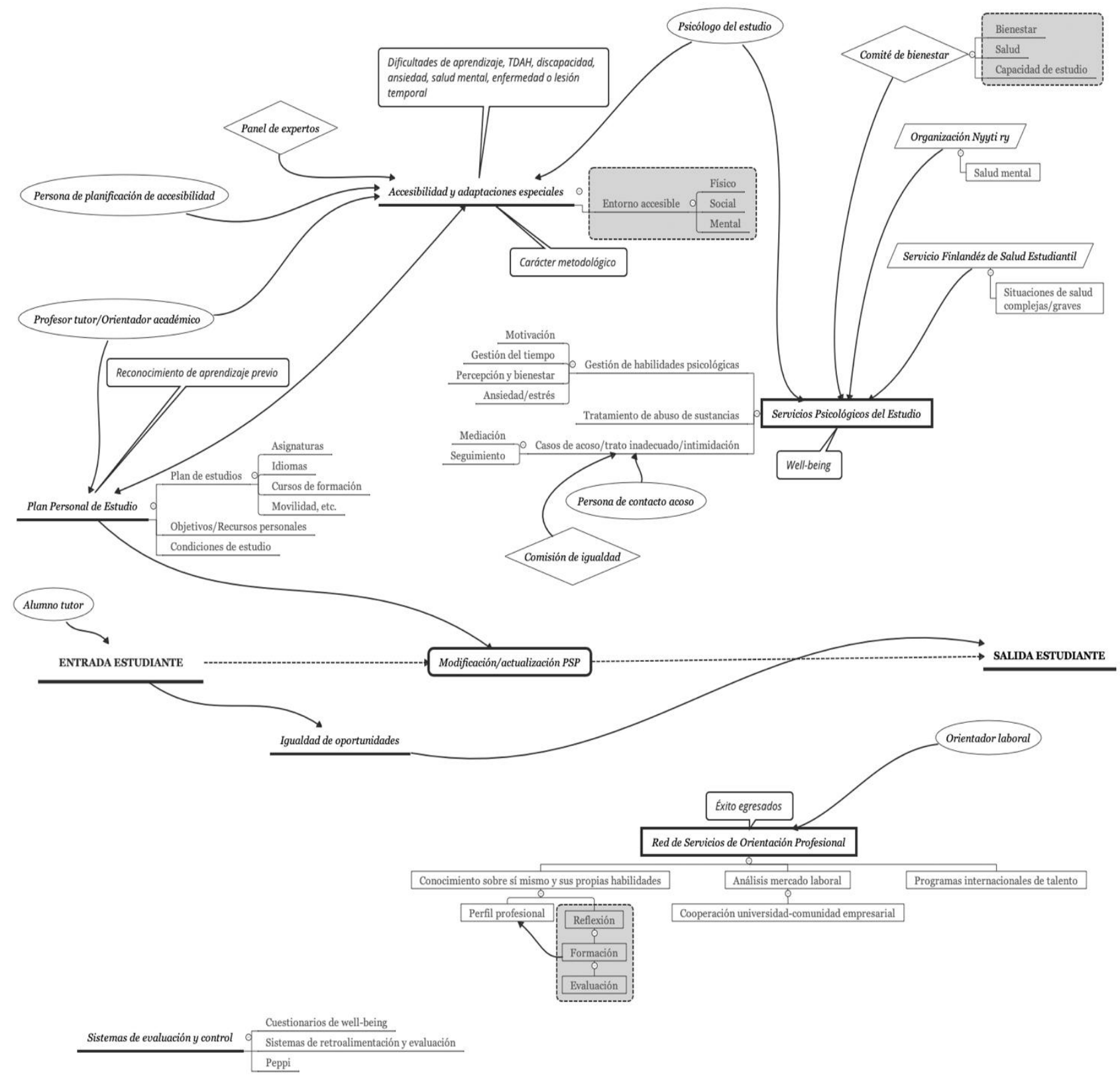

